\title{
Angle-dependent modulated spectral peaks of proton beams generated in ultrashort intense laser-solid interactions
}

\author{
L. N. Su, ${ }^{1}$ Z. D. Hu, ${ }^{1}$ Y. Zheng, ${ }^{1}$ M. Liu, ${ }^{1}$ Y. T. Li, ${ }^{1, a)}$ W. M. Wang, ${ }^{1}$ Z. M. Sheng ${ }^{2,3}$ X. \\ H. Yuan, ${ }^{2}$ M. H. Xu, ${ }^{4}$ Z. W. Shen, ${ }^{1}$ H. T. Fan, ${ }^{1}$ L. M. Chen,,${ }^{1}$ X. Lu, ${ }^{1}$ J. L. Ma, ${ }^{1}$ X. \\ Wang $^{1}$, Z. H. Wang, ${ }^{1}$ Z. Y. Wei, ${ }^{1}$ and J. Zhang ${ }^{2 \text { b) }}$
}

${ }^{I}$ Beijing National Laboratory for Condensed Matter Physics, Institute of Physics, Chinese Academy of Sciences, Beijing 100190, China

${ }^{2}$ Key Laboratory for Laser Plasmas (MoE) and Department of Physics and Astronomy, Shanghai Jiao Tong University, Shanghai 200240, China

${ }^{3}$ Department of Physics, SUPA, University of Strathclyde, Glasgow G4 ONG, UK

${ }^{4}$ Department of Physics, China University of Mining and Technology (Beijing), Beijing 100083, China

Proton acceleration from $4 \mu \mathrm{m}$ thick aluminum foils irradiated by 30-TW Ti: sapphire laser pulses are investigated using an angle-resolved proton energy spectrometer. We find that a modulated spectral peak at $\sim 0.82 \mathrm{MeV}$ is presented at $2.5^{\circ}$ off the target normal direction. The divergence angle of the modulated zone is $3.8^{\circ}$. Two-dimensional particle-in-cell simulations reveal that self-generated toroidal magnetic field at the rear surface of the target foil is responsible for the modulated spectral feature. The field deflects the low energy protons, resulting in the modulated energy spectrum with certain peaks.

a) Electronic mail: ytli@aphy.iphy.ac.cn;

b) Electronic mail: jzhang1@sjtu.edu.cn 


\section{INTRODUCTION}

Laser-driven ion beams hashave advantages of short pulse duration, high brightness and small source size. For their potential applications such as proton radiography ${ }^{1}$, proton-driven fast ignition ${ }^{2}$, tumour therapy ${ }^{3}$, proton-driven nuclear reactions ${ }^{4}$, etc., monoenergetic spectral distributions of protons are preferred. However, most of the experimentally generated proton beams present exponential-like proton energy spectra. To produce proton beams with modulated spectral distributions, several mechanisms, such as radiation pressure acceleration ${ }^{5}$, break-out afterburner ${ }^{6}$, and laser-driven shock acceleration ${ }^{7}$, have been proposed, and successfully demonstrated by numerical simulations and experiments ${ }^{8,9}$. However, to implement these mechanisms the drive laser pulses must have very high contrast ratio better than $10^{-10}$ and high focused intensity higher than $10^{21} \mathrm{~W} / \mathrm{cm}^{2}$, typically. Such requirements are great challenges for the laser systems in commission.

With the current laser parameters, the target normal sheath acceleration (TNSA) has been the typical mechanism found in experiments for laser-driven ion acceleration ${ }^{10,11}$. How to generate proton beams with modulated spectral distributions by TNSA mechanism has been investigated by many groups in the last decade. In the interaction of a 20-TW/0.8 ps laser with a hydrogen-desorbed palladium foil target, Hegelich et al. obtained a spectrally modulated $\mathrm{C}^{5+}$ ion beam by removing the contaminants on the target rear surface $^{12}$. Schwoerer et al. reported quasi-monoenergetic protons emitted in the target normal direction from microstructured targets with a $10 \mathrm{TW}$ Ti: sapphire $\operatorname{laser}^{13}$. Toncian et al. used ultrafast laser-driven micro lens to focus $\mathrm{MeV}$ protons with specific energies, generating modulated proton beams ${ }^{14}$.

Based upon the target manipulation, Robinson et al. observed quasi-monoenergetic proton beams in the target normal direction from 50 200 nm planar foil targets irradiated by the ASTRA Ti: sapphire laser

pulses ${ }^{15}$. From particle-in-cell (PIC) simulations, they find two self-generated toroidal magnetic fields at the rear surface of the $\sim \mathrm{nm}$ ultrathin foil. The field near the target center will focus protons, while the field a little far from the target center will deflect protons off the target normal direction. According to their explanation, the focusing field can self-select protons with certain energies, leading to the spectral peaks. For micron-thickness targets, the focusing field will disappear and no spectral peaks are observed in the 
normal direction.

In this paper, we will present our observation of proton beams emitted from micron-thickness foils irradiated by 30-TW Ti: sapphire laser pulses by using an angle-resolved magnetic spectrometer. We find that modulated spectral peaks are presented at $2.5^{\circ}$ off the target normal direction for $4 \mu \mathrm{m}$ thick aluminum foil targets. Our PIC simulations show that a strong toroidal magnetic field is also generated at the rear target surface. This field will deflect (rather than focus) the low energy protons away and make the protons with certain energies outstanding.

\section{EXPERIMENTAL SETUP}

The experiment was conducted on the Xtreme Light (XL) Ti: sapphire laser facility at the Institute of Physics, Chinese Academy of Sciences ${ }^{16}$. The schematic setup is shown in Fig 1. A p-polarized laser pulse of $100 \mathrm{fs}$ pulse duration, $1 \sim 2.5 \mathrm{~J}$ energy and $-800 \mathrm{~nm}$ central wavelength was focused using an $f / 1.67$ off-axis parabola (OAP) mirror onto target foils at an incidence angle of $15^{\circ}$. The laser energy on target after compression was from 1 to $2.5 \mathrm{~J}$. The full width at half maximum (FWHM) of the laser focal spot was $8 \mu \mathrm{m}$, giving a maximum laser intensity of $5 \times 10^{19} \mathrm{~W} / \mathrm{cm}^{2}$. The contrast ratio at 7 nanoseconds before the main laser pulse was better than $10^{-6}$. The targets were $4 \mu \mathrm{m}$-thick aluminum foils.

An angle-resolved proton energy spectrometer ${ }^{17}$ was set at $8 \mathrm{~cm}$ away from the target. A $30 \mathrm{~mm} \times 0.2$ $\mathrm{mm}$ horizontal lead slit was used as an entrance of the spectrometer. A slowly varying magnetic field with central field strength of $0.3 \mathrm{~T}$ was applied to disperse the protons. The spectrometer could collect the protons emitted into the angle range of $16^{\circ}$, which covered both the laser propagation (LP) direction and target normal (TN) direction. The vertical collection angle was $2.5 \mathrm{mrad}$. The detector was $98 \mathrm{~mm} \times 49 \mathrm{~mm}$ calibrated TR-imaging plates (IPs). The resolution of the IP scanner is $50 \mu \mathrm{m}$. The angular resolution of the spectrometer was $0.14^{\circ}$, determined by the slit width and the scanner resolution. A $4 \mu \mathrm{m}$ thick aluminum filter was used to cover the imaging plates to block the carbon ions with energies less than $4 \mathrm{MeV}$. In the present experiment, we found that the carbon ions with energies higher than $4 \mathrm{MeV}$ were not measurable. 
This was confirmed by using a traditional Thomson parabolic spectrometer with a pinhole as an entrance.

\section{RESULTS AND DISCUSSIONS}

A typical proton image on the imaging plate obtained for a $4 \mu \mathrm{m}$-thick Al target is shown in Fig.2 (a). The red line on the top of the image is formed by photons and neutral particles directly through the entrance slit, which can be used as the original points for the proton spectra. The vertical and horizontal directions correspond to the proton energy dispersion and angular distribution, respectively. We define $\theta$ to be the angle respect to the target normal direction. $\theta=15^{\circ}$ corresponds to the laser propagation direction. Note that the large distance between the two magnets leads to the magnetic field profile to be a Gaussian-like distribution, which results in the curved top edge of the proton image. This has been corrected when deducing proton spectra.

From Fig. 2(a), we can see the proton spectra are angle-dependent. We plot the proton spectra in the target normal direction $\left(\theta=0^{\circ}\right)$ and laser propagation $\left(\theta=15^{\circ}\right)$ in Fig.2 (b) and (c), respectively. For the target normal direction, the proton cut-off energy is $1.2 \mathrm{MeV}$ and the temperature is $0.23 \mathrm{MeV}$. For the laser propagation direction, the proton cut-off energy is $1.26 \mathrm{MeV}$, and the average temperature is $0.31 \mathrm{MeV}$. There are no modulated peaks presented in both directions.

An interesting feature in Fig.2 (a) is the presence of a yellow ellipse in the energy range from 0.51 to $0.75 \mathrm{MeV}$ and the angle range from $0.5^{\circ}$ to $4.3^{\circ}$ off the target normal direction, marked by a black dashed circle. Proton counts per unit area in the yellow ellipse are obviously less than those in the surrounding regions, so that there should be spectrally modulated distributions presented around the ellipse. The proton energy spectra in the middle $\left(\theta=2.5^{\circ}\right)$ and the right side $\left(\theta=1.2^{\circ}\right)$ of the ellipse are shown in Fig.2 (d) and (e), respectively. For the middle position, the count of protons with energies of less than $0.76 \mathrm{MeV}$ is obviously less than that in the target normal direction, resulting in the protons at $\sim 0.82 \mathrm{MeV}$ outstanding in the spectrum. The $\Delta E / E$ of the modulated spectral peak is estimated to be $25 \%$. For the right side of the ellipse, the modulated spectral peak is at $0.76 \mathrm{MeV}$ and the $\Delta E / E$ is about $33 \%$. For $2.5^{\circ}$, the proton count 
at the valley $(E=0.71 \mathrm{Mev})$ is $3.49 \times 10^{5}$; while the proton count at the peak $(E=0.82 \mathrm{Mev})$ is $6.65 \times 10^{5}$. As a result, the depth of the modulation $\Delta N / N$ is $48 \%$. Meanwhile, for $1.2^{\circ}$, the depth of modulation is $43 \%$.

Ultrafast laser-driven proton radiography shows that a large amplitude (thousands of Tesla) magnetic field is generated at the rear of foil targets irradiated by high-intensity laser pulse ${ }^{18}$. Robinson et al. has found that the self-generated magnetic field plays important role on the presence of the quasi-monoenergetic proton peaks for ultrathin foils ${ }^{15}$. To understand our observation for thick targets, we have carried out numerical simulations by the $2 \mathrm{D} 3 \mathrm{~V}$ PIC code $\mathrm{KLAPS}^{19,20}$. The simulation parameters are similar to the experimental. The temporal and spatial resolutions in the simulations are $\mathrm{d} t=0.025 \tau_{0}$, and $\mathrm{d} x=\mathrm{d} y=0.025 \lambda_{0}$, where $\lambda_{0}$ and $\tau_{0}$ are laser wavelength and laser period, respectively. There are 36 simulation particles in each cell, for both ions and electrons. Absorption boundaries for the fields and particles have been adopted. A Gaussian laser pulse with a normalized vector potential $a_{0}=2.4$ (corresponding to the laser intensity of $2.5 \times 10^{19} \mathrm{~W} / \mathrm{cm}^{2}$ ) is incident from the left boundary at an angle of $15^{\circ}$. The target is located at the region $40 \lambda_{0} \leqslant x \leqslant 50 \lambda_{0},-35 \lambda_{0} \leqslant y \leqslant 35 \lambda_{0}$. The target consists of a uniform plasma slab with a density of $n_{e}=30 n_{c}$ and a preplasma, where $n_{c}$ is the plasma critical density. The preplasma density profile at the front surface of target follows $n_{e}(x)=1.5 \gamma_{o s c} n_{c} \exp \left[(x-40) / 2 \lambda_{L}\right]$, where $\gamma_{o s c}=\sqrt{1+a_{0}^{2} / 2}$ is the Lorentz factor related to the quiver oscillation of electrons driven by the laser electric field. The preplasma scale length $\lambda_{L}$ is set to be $2 \lambda_{0}$.

The simulated proton spectra emitted into a collection angle of $2.5 \mathrm{mrad}$ in the direction of $\theta=2.5^{\circ}$ from the target normal are plotted, as shown in Fig. 3. We can see a modulated spectral peak presented at $0.81 \mathrm{MeV}$. This is in good agreement with the observed spectrum in Fig. 2(d). In the simulations, a self-generated toroidal magnetic field with strengths $100 \sim 1000 \mathrm{~T}$ is observed at the rear surface. We plot distributions of the simulated magnetic fields and the electrostatic fields in Fig.4. According to the Maxwell Equations, $\dot{B}_{z} \approx \partial_{y} E_{x}$, the target normal sheath field varies transversely with time, generating the toroidal magnetic field in the rear surface of target ${ }^{15}$. The magnetic field is symmetrical at $100 \tau_{0}$, and then becomes more and more asymmetrical at later time. This is associated the asymmetric electron generation and transport with oblique incidence of the laser pulse. 
We track the trajectories of $1 \%$ of the total protons with energies higher than $0.2 \mathrm{MeV}$ in the simulations. Their trajectories in momentum space are plotted in Fig. 4, where $P_{x}$ and $P_{y}$ are the longitudinal and transverse momentum, respectively. The blue lines correspond to the $E>0.8 \mathrm{MeV}$ protons, and the black lines to the $0.2 \sim 0.8 \mathrm{MeV}$ protons. The $E<0.8 \mathrm{MeV}$ protons have large transverse momentum. However, the transverse momentum of the $E>0.8 \mathrm{MeV}$ protons is much lower than the longitudinal momentum. This indicates that the low energy protons are much scattered and the high energy protons are directional. The experimental angle-dependent spectral peaks are believed to be caused by the deflection of the protons due to the self-generated magnetic field. Under the influence of the field, most of the $E<0.8$ $\mathrm{MeV}$ protons are deflected out of the collection angle of $2.5 \mathrm{mrad}$, while the $E>0.8 \mathrm{MeV}$ protons are not. Therefore, the counts of the $E>0.8 \mathrm{MeV}$ protons are outstanding in the spectrum.

In previous experiments by Robinson et al. ${ }^{15}$, a quasi-monoenergetic proton peak is observed within a collection angle of $0.5 \mathrm{mrad}$ in the target normal direction by using ultrathin nm-thick targets. The focusing effect of the self-generated magnetic field near the target center is responsible for the quasi-monoenergetic peaks. While, for $\mu \mathrm{m}$-thick targets no monoenergetic feature is observed in the target normal. In our experiments, we have observed modulated spectral peaks off target normal direction for micron-thick targets. This indicates that the deflection effect of the self-generated magnetic field can also work on the formation of modulated spectral peaks in some directions.

\section{CONCLUSIONS}

We have observed proton beams with spectral peaks presented in off-target normal direction in the interactions of femtosecond laser pulses with micron-thickness foil targets. Our PIC simulations show that a 100 1000 T toroidal magnetic field is generated at the rear target surface. The field can deflect most of protons, particularly for those with relatively low energy protons, consequently generating modulated spectral peaks. The peaks may be optimized by controlling the self-generated magnetic field through changing laser and target conditions. 


\section{Acknowledgements}

This work was supported by National Basic Research Program of China (Grant No.2013CBA01501) and the National Nature Science Foundation of China (Grant Nos. 11375262, 11135012).

Reference:

${ }^{1}$ M. Borghesi, D. H. Campbell, A. Schiavi, M. G. Haines, O. Willi, A. J. MacKinnon, P. Patel, L. A. Gizzi, M. Galimberti, R. J. Clarke, F. Pegoraro, H. Ruhl, and S. Bulanov, Phys. Plasmas 9, 2214 (2002).

${ }^{2}$ M. Roth, T. E. Cowan, M. H. Key, S. P. Hatchett, C. Brown, W. Fountain, J. Johnson, D. M. Pennington, R. A. Snavely, S. C. Wilks, K. Yasuike, H. Ruhl, F. Pegoraro, S. V. Bulanov, E. M. Campbell, M. D. Perry, and H. Powell, Phys. Rev. Lett. 86, 436 (2001).

${ }^{3}$ S. V. Bulanov and V. S. Khoroshkov, Plasma Phys. Rep.28, 453 (2002).

${ }^{4}$ K. W. D. Ledingham, P. McKenna, R. P. Singhal, Science, 300, 1107 (2003).

${ }^{5}$ A. Henig, S. Steinke, M. Schnürer, T. Sokollik, R. Hörlein, D. Kiefer, D. Jung, J. Schreiber, B. M.

Hegelich, X. Q. Yan, J. Meyer-ter-Vehn, T. Tajima, P. V. Nickles, W. Sandner, and D. Habs, Phys. Rev.

Lett. 103, 245003 (2009).

${ }^{6}$ L. Yin, B. J. Albright, B. M. Hegelich, K. J. Bowers, K. A. Flippo, T. J. T. Kwan and J. C. Fernández,

Phys. Plasmas 14, 056706 (2007)

${ }^{7}$ A. Henig, D. Kiefer, M. Geissler, S. G. Rykovanov, R. Ramis, R. Hörlein, J. Osterhoff, Zs. Major, L.

Veisz, S. Karsch, F. Krausz, D. Habs, and J. Schreiber, Phys. Rev. Lett. 102, 095002 (2009)

${ }^{8}$ B M Hegelich, I Pomerantz, L Yin, H C Wu, D Jung, B J Albright, D C Gautier, S Letzring, S

Palaniyappan, R Shah, K Allinger, R Hörlein, J Schreiber, D Habs, J Blakeney, G Dyer, L Fuller, E Gaul, E 
Mccary, A R Meadows, C Wang, T Ditmire and J C Fernandez, New J. Phys. 15085015 (2013)

${ }^{9}$ Dan Haberberger, Sergei Tochitsky, Frederico Fiuza, Chao Gong, Ricardo A. Fonseca, Luis O. Silva,

Warren B. Mori \& Chan Joshi, Nature Physics 8, 95-99 (2012)

${ }^{10}$ M. H. Xu, Y. T. Li, X. H. Yuan, Q. Z. Yu, S. J. Wang, W. Zhao, X. L. Wen, G. C. Wang, C. Y. Jiao, Y. L.

He, S. G. Zhang, X. X. Wang, W. Z. Huang, Y. Q. Gu and J. Zhang, Phys. Plasmas 13, 104507 (2006)

${ }^{11}$ Y. Q. Cui, W. M. Wang, Z. M. Sheng, Y. T. Li and J. Zhang, Phys. Plasmas 20, 024502 (2013)

${ }^{12}$ B. M. Hegelich, B. J. Albright, J. Cobble, K. Flippo, S. Letzring, M. Paffett, H. Ruhl, J. Schreiber, R. K.

Schulze \& J. C. Fernandez, Nature 439, 04400 (2006).

${ }^{13}$ H. Schwoerer, S. Pfotenhauer, O. Jackel, K.-U. Amthor, B. Liesfeld, W. Ziegler, R. Sauerbrey, K. W. D.

Ledingham\& T. Esirkepov, Nature 439, 04492 (2006).

${ }^{14}$ T. Toncian, M. Borghesi, J. Fuchs, E. d'Humieres, P. Antici, P. Audebert, E. Brambrink, C. A. Cecchetti, A. Pipahl, L. Romagnani, O. Willi, Science 312, 410 (2006).

${ }^{15}$ A. P. L. Robinson, P. Foster, D. Adams, D. C. Carroll, B. Dromey, S. Hawkes, S. Kar, Y. T. Li, K.

Markey, P. McKenna, C. Spindloe, M. Streeter, C.-G. Wahlström, M. H. Xu, M. Zepf and D. Neely, New J.

Phys. 11, 083018 (2009).

${ }^{16}$ Z. H. Wang, C. Liu, Z. W. Shen, Q. Zhang, H. Teng, and Z. Y. Wei, Opt. Lett. 36, 3194 (2011).

${ }^{17}$ Y. Zheng, L. N. Su, M. Liu, B. C. Liu, Z. W. Shen, H. T. Fan, Y. T. Li, $\quad$ L. M. Chen, X. Lu, J. L. Ma, W. M. Wang, Z. H. Wang, Z. Y. Wei and J. Zhang, Rev. Sci. Instrum. 84, 096103 (2013).

${ }^{18}$ G. Sarri, A. Macchi, C. A. Cecchetti, S. Kar, T.V. Liseykina, X. H. Yang, M. E. Dieckmann, J. Fuchs, M.

Galimberti, L. A. Gizzi, R. Jung, I. Kourakis, J. Osterholz, F. Pegoraro, A. P. L. Robinson, L. Romagnani, O. Willi, and M. Borghesi, Phys. Rev. Lett. 109, 205002 (2012). 
${ }^{19}$ M. Chen, Z. M. Sheng, J. Zheng, Y. Y. Ma, and J. Zhang, Chin. J. Comput. Phys. 25, 43 (2008);

${ }^{20}$ W. M. Wang, Z.M. Sheng, P. A. Norreys, M. Sherlock, R. Trines, A. P. L. Robinson, Y. T. Li, B. Hao, and J. Zhang, J. Phys.: Conf. Ser. 244, 022070 (2010).

\section{Figure Captions}

FIG. 1. (Color online)Experimental schematic. A $p$-polarized laser pulse was focused by an f/1.67OAP mirror onto a $4 \mu \mathrm{m}$ aluminum foil at an incidence angle of $15^{\circ}$. The FWHM of the laser focal spot is $8 \mu \mathrm{m}$, giving the maximum laser intensity up to $5 \times 10^{19} \mathrm{~W} / \mathrm{cm}^{2}$. The protons generated from the target are measured by an angle-resolved proton energy spectrometer. Using a horizontal lead slit as an entrance, the spectrometer can collect the protons within the laser propagation direction and the target normal direction.

FIG. 2. (Color online) Raw image of proton signal on the IP detector (a) and the lineout proton spectra in different directions (b-e). Laser energy on target is obtained at $1.9 \mathrm{~J}$. The horizontal and vertical directions of the image correspond to the angular distribution and the energy dispersion of the protons, respectively. Note that there is a yellow ellipse area from $0.5^{\circ}$ to $4.3^{\circ}$ where the proton numbers are obviously lower than the surrounding region. This area is marked with a black dashed elliptic circle in (a). The region above the ellipse, marked by a solid black box, shows a spectrally modulated feature. ( $\mathrm{b}-\mathrm{e}$ ) are the proton spectra for the four typical directions, $\theta=0^{\circ}$ (the target normal), $15^{\circ}$ (the laser propagation), $2.5^{\circ}$ (the middle of the ellipse) and $1.2^{\circ}$ (the side of the ellipse), respectively. The insets in (d) and (e) show the spectral peaks in linear ordinate. Proton counts detected by the IP are given per $1 \mathrm{MeV}$ and per $6 \times 10^{-3} \mathrm{msr}$. Spectral peaks at $0.82 \mathrm{MeV}$ in (d), and at $0.76 \mathrm{MeV}$ in (e) are presented, while no significant spectral peaks in (b) and (c).

FIG. 3. Simulated spectrum of the protons emitted at $<2.5 \mathrm{mrad}$ in the direction of $\theta=2.5^{\circ}$ from the target normal at $t=300 \tau_{0}$. A spectral peak at $0.81 \mathrm{MeV}$ is presented. 
FIG. 4. (Color online) Distributions of the simulated magnetic fields, $B_{z}$, at $t=100 \tau_{0}$ (a), $t=200 \tau_{0}$ (c), and $t=300 \tau_{0}$ (e) and the normalized electrostatic fields, $E_{x}$, at $t=100 \tau_{0}(\mathrm{~b}), t=200 \tau_{0}(\mathrm{~d})$, and $t=300 \tau_{0}(\mathrm{f})$. The target is located at the region $40 \leq x / \lambda_{0} \leq 50,-35 \leq y / \lambda_{0} \leq 35$. The magnetic field is in unit of Tesla. The magnetic field strength is up to 1000T.

FIG. 5. (Color online) Proton trajectories in momentum space in the simulation. $P_{x}$ and $P_{y}$ are normalized proton longitudinal and transverse momentum, respectively. The blue lines correspond to the $E>0.8 \mathrm{MeV}$ protons, while the black lines to the $E<0.8 \mathrm{MeV}$ protons. $E<0.8 \mathrm{MeV}$ protons have much higher transverse momentums than that for $E>0.8 \mathrm{MeV}$ protons. 


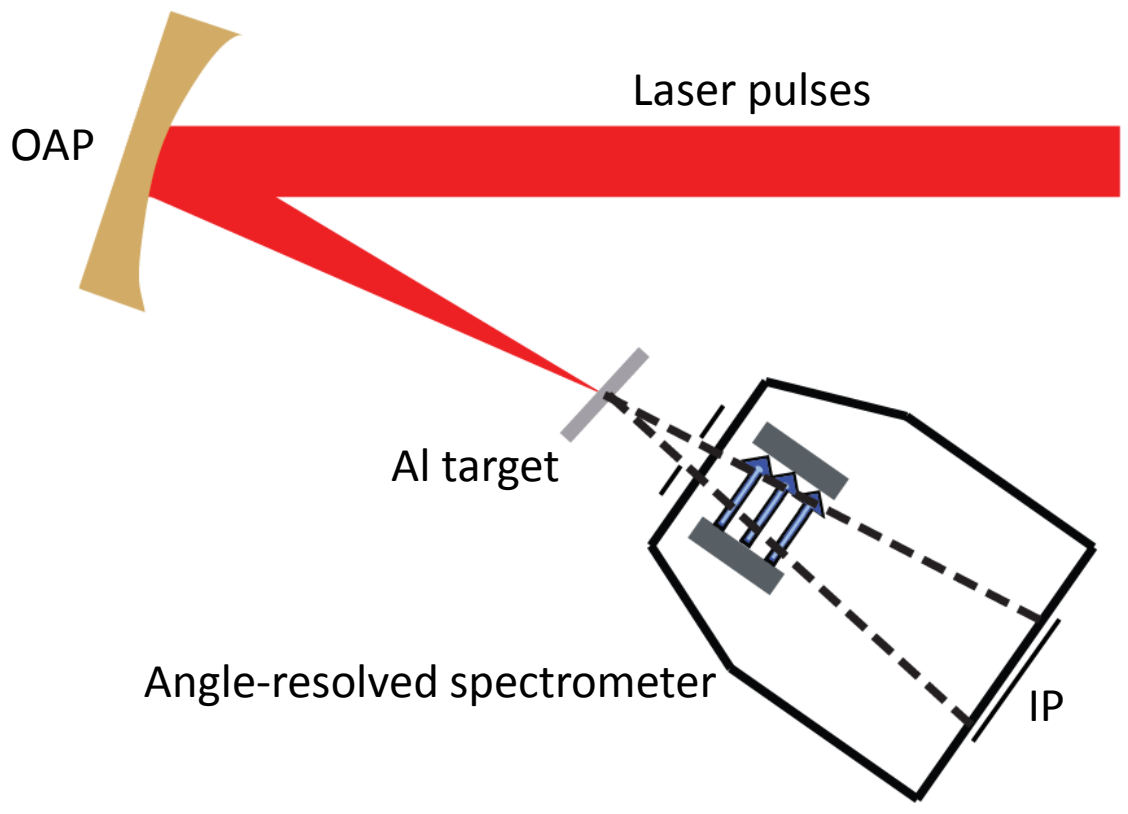

Figure 1 


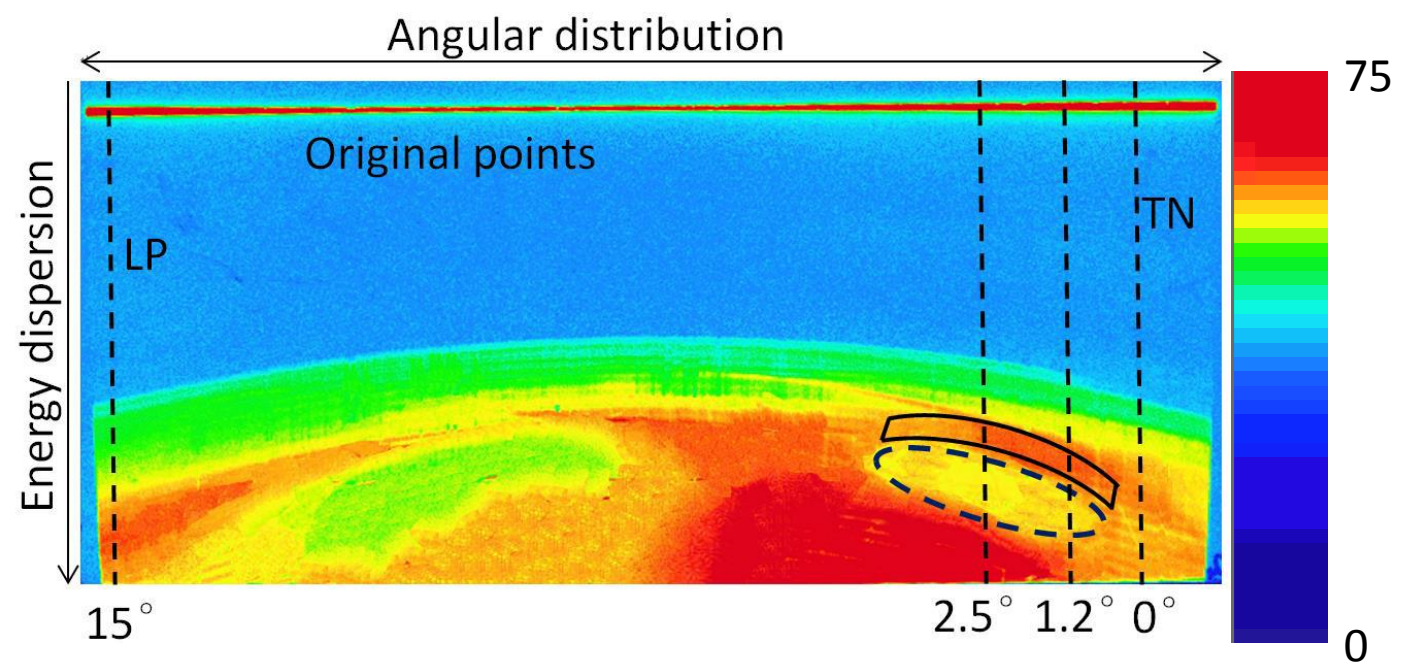

(a)

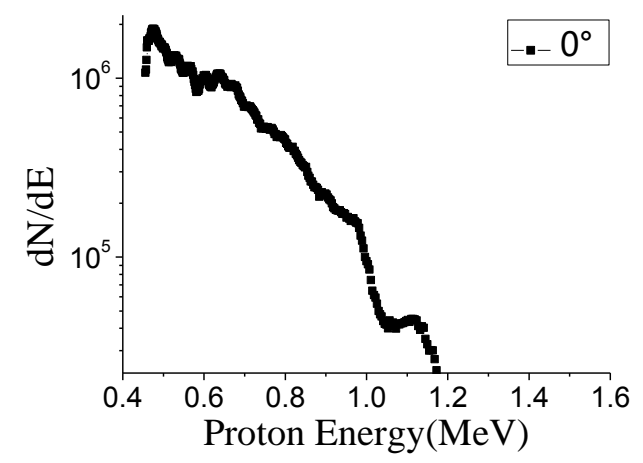

(b)

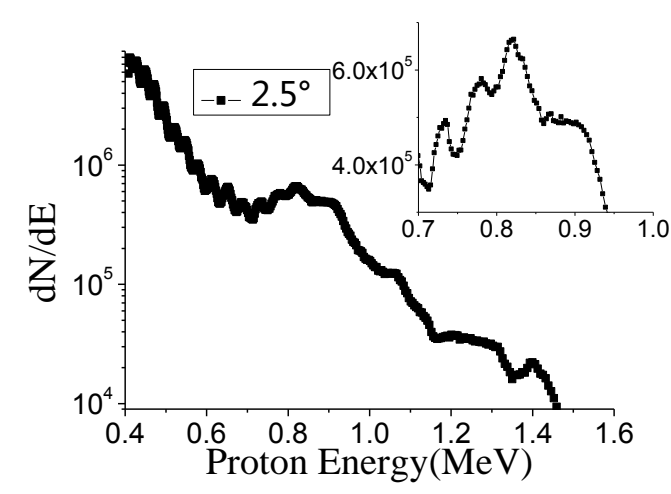

(d)

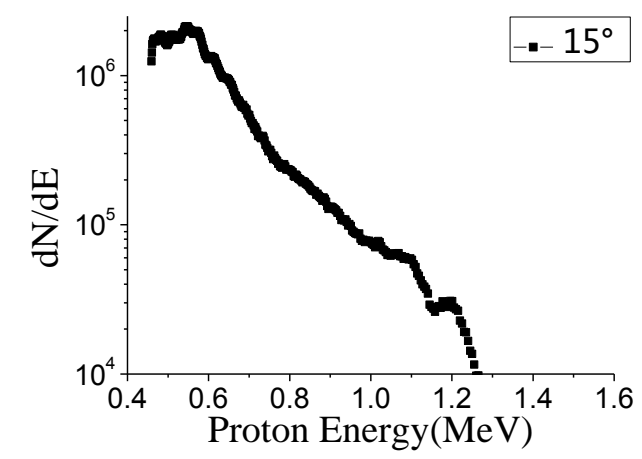

(c)

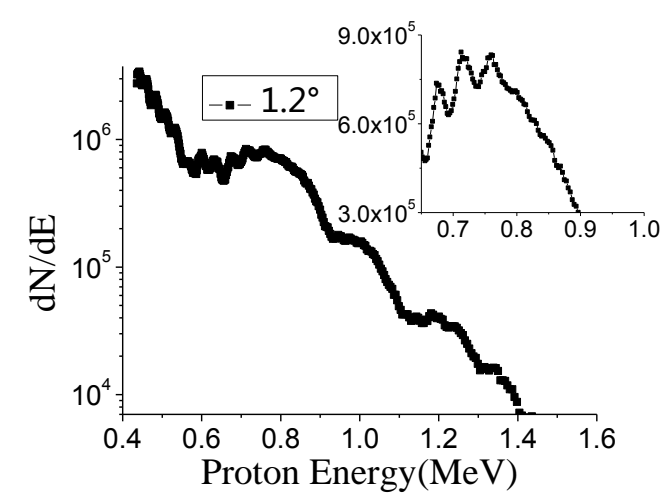

(e)

Figure 2 

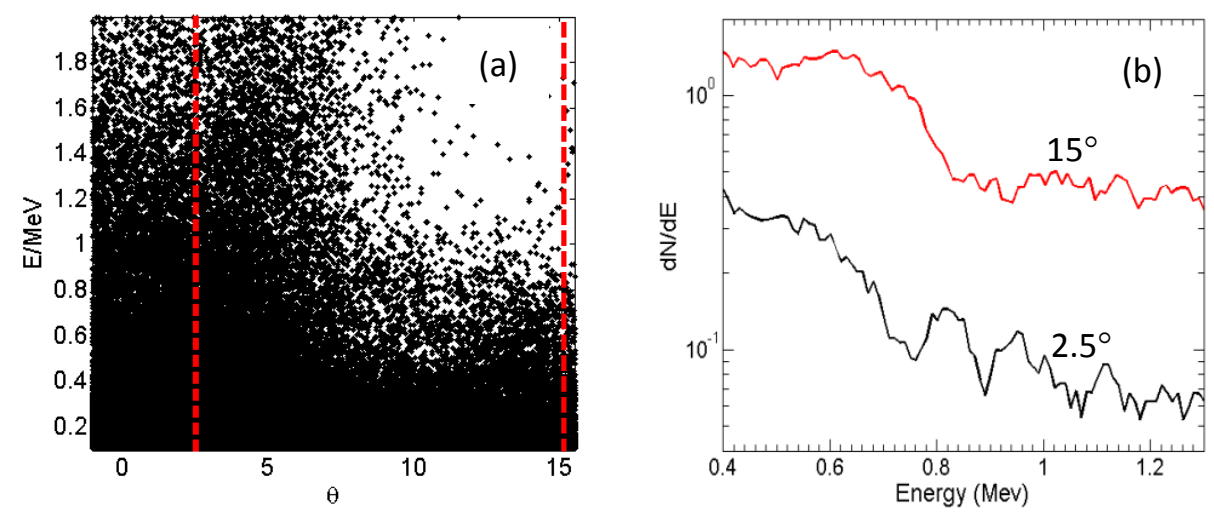

Figure 3 


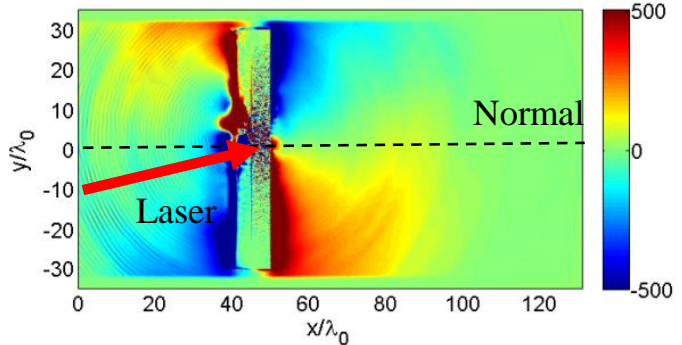

(a)

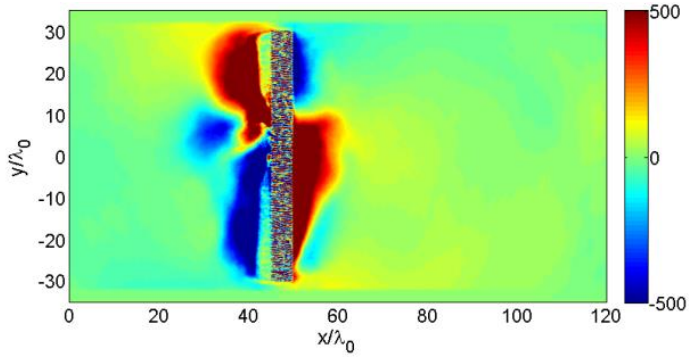

(c)

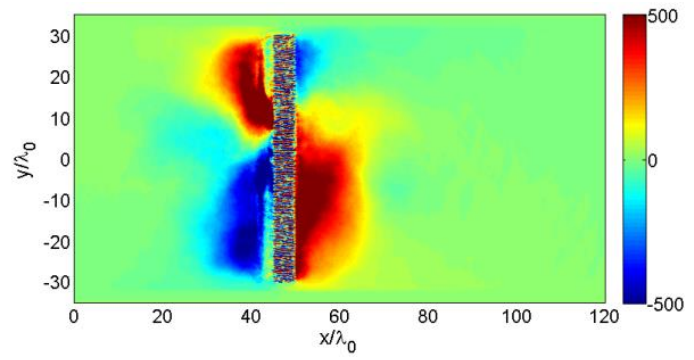

(e)

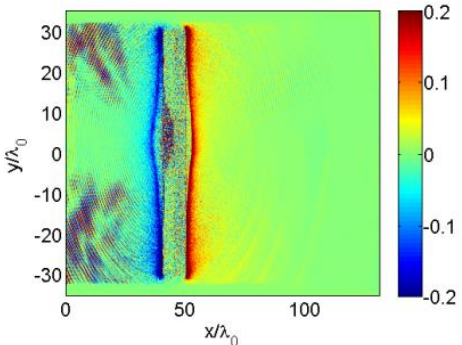

(b)

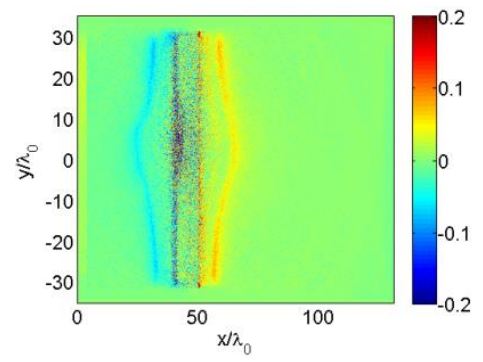

(d)

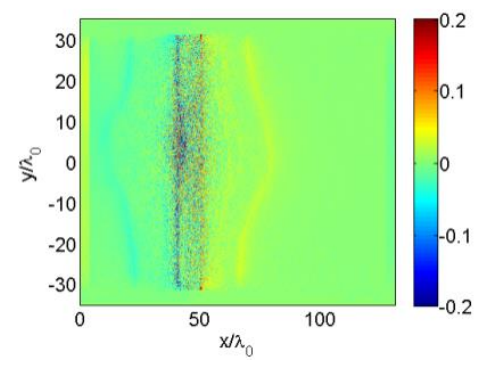

(f)

Figure 4 


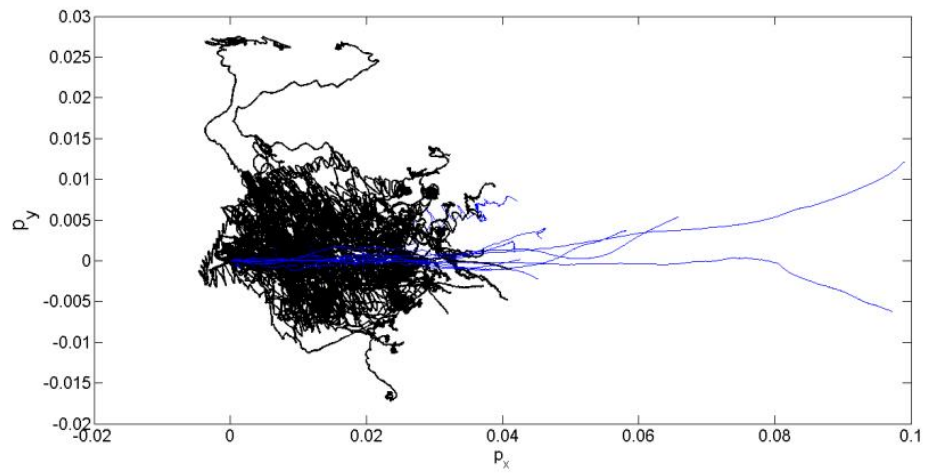

Figure 5 